\title{
Correction to "A theorem on finite projective planes of odd order and an application to planes of order 15"
}

\author{
By \\ Vlado Cigić
}

In the formulation of the Theorem 1 of the above-mentioned paper, appeared in Arch. Math. 41, 280-288 (1983), there was a mistake, which can be easily corrected. Instead of " $p$ an odd prime" it should read "where $p>3$ is a prime number."

Eingegangen am 17.9.1984

Anschrift des Autors:

Vlado Cigić

Gradjevinski fakultet Mostar

79000 Mostar

Yugoslavia 